\title{
BACKWARD BIFURCATION IN A MATHEMATICAL MODEL OF PI3K/AKT PATHWAYS IN ACUTE MYELOID LEUKEMIA
}

\author{
Yudi Ari Adi ${ }^{1,2, *}$, Lina Aryati $^{2}$, Fajar Adi-Kusumo ${ }^{2}$ and \\ Mardiah Suci Hardianti ${ }^{3}$ \\ ${ }^{1}$ Department of Mathematics \\ Faculty of Applied Science and Technology \\ Universitas Ahmad Dahlan \\ Yogyakarta 55191, Indonesia \\ ${ }^{2}$ Department of Mathematics \\ Faculty of Mathematics and Natural Sciences \\ Universitas Gadjah Mada \\ Yogyakarta 55281, Indonesia \\ ${ }^{3}$ Department of Internal Medicine \\ Faculty of Medicine, Public Health and Nursing \\ Universitas Gadjah Mada \\ Yogyakarta 55281, Indonesia
}

\begin{abstract}
This paper investigates a mathematical model of the PI3K/AKT pathway in acute myeloid leukemia (AML) in the absence of protein dephosphorylation and AKT degradation. We perform a bifurcation analysis by using the bifurcation method, which is based on the use of
\end{abstract}

Received: July 9, 2019; Accepted: August 5, 2019

2010 Mathematics Subject Classification: 34C23, 62P10.

Keywords and phrases: AML, mathematical model, backward bifurcation.

${ }^{*}$ Corresponding author 
the center manifold theory. We give an explicit condition for the existence of backward and forward bifurcations. Numerical simulations are presented to support analytical results and then discussed from both the mathematical and the medical perspectives.

\section{Introduction}

Phosphoinositide 3-kinase (PI3K)/protein kinase B (PKB) also known as AKT is an important mediator in the regulation of hematopoiesis. Constitutive activation of PI3K/AKT signaling pathway has been observed in acute myeloid leukemia (AML), characterized by abnormal differentiated and uncontrolled proliferation of immature myeloid cells [1]. FMS-like tyrosine kinase (FLT3) mutation is the most frequent molecular abnormality in AML that causes constitutive activation of the PI3K/AKT pathway. AML is the most common myeloid leukemia in adults that have a poor clinical outcome, with only about $10 \%$ of patients survive. The treatment of AML mainly based on standard chemotherapy with or without stem cell transplantation has changed little in the recent decade, among with the development of small molecules that target the disease on a molecular level [2-4]. Recently, the US Food and Drug Administration (FDA) approved midostaurin to treat FLT3 mutated AML [5]. Midostaurin is a small molecule inhibitor that targets FLT3 which is used in combination with chemotherapy. Currently, numerous agents are under development for the treatment of AML as do a novel combination with FLT3 inhibitors, such as quizartinib, gilteritinib, and selinexor $[2,5]$. Therefore, it is important to understand the most important parameters in the PI3K/AKT pathway, which have significance in the AML diseases and should be intervened.

Mathematical modeling in the PI3K/AKT pathway can help improve our understanding of molecular dynamics in this pathway. Motivated by several recent studies, numerous new agents are under development to treat AML patients. In previous work, we have developed a mathematical model that describes the interaction of the protein in the PI3K/AKT pathway on AML [6]. This modeling result suggested the potential protein in the PI3K/ AKT 
pathway that should be targeted to AML therapy. In this paper, we propose a new model by ignoring the protein dephosphorylation and AKT degradation rate. In this model, we consider the fact that in AML, the mechanism of protein dephosphorylation does not proceed normally, and the degradation processes of AKT relatively slower than the AKT phosphorylation [7]. Furthermore, in this study, we will perform a qualitative analysis and indicate that the model undergoes a backward bifurcation by using the method introduced by Castillo-Chavez and Song in [8].

In a medical point of view, the occurrence of backward bifurcation plays an important role in disease control and eradication. In a compartment epidemic model, it has been widely accepted that the condition of basic reproduction number $R_{0}$ is an essential requirement for the elimination of diseases. If $R_{0}<1$, then the disease-free equilibrium is asymptotically stable and infection dies out, whereas if $R_{0}>1$, then the endemic equilibrium point is asymptotically stable and the infection persists. However, the phenomenon of backward bifurcation causes the $R_{0}<1$ condition not to be fully adequate as a condition for eradicating the disease. The backward bifurcation scenario involves a stable disease-free equilibrium coexists with a stable and unstable endemic equilibrium. Medically, the backward bifurcation at $R_{0}=1$ makes treatment or control of diseases more difficult than that of the model which exhibits forward bifurcation. In the case of forward bifurcation, the infection or diseases usually do not persist whenever $R_{0}<1$. Therefore, the occurrence of backward bifurcation has important implications in many infectious diseases. It has been observed in several recent studies, either for generic or specific diseases, see [9-14]. Bifurcation analysis in these studies was carried out based on the use of center manifold theory $[8,15]$.

Such as in epidemic modeling, in this paper, we aim to provide the bifurcation threshold and derive conditions in term of parameters of the system to ensuring the occurrence of backward or forward bifurcation. The paper is organized as follows. A model of PI3K/AKT pathway in acute 
myeloid leukemia is formulated and analyzed in Section 2. In Section 3, we determine threshold values and derive sufficient conditions for the scenario of backward or forward bifurcation. In Section 4, we provide some numerical simulations to verify the theoretical results obtained in Section 3. We then make a conclusion and discussion of this study in Section 5.

\section{Model Formulation}

In this paper, we improved our previous model in [6] by adding assumption that FOXO3a as a transcriptional regulator always exists in a certain amount within the nucleus [16]. Then we consider the simplified model by ignoring the protein dephosphorylation and AKT degradation rate. This is due to the fact that in AML the mechanism of protein dephosphorylation does not proceed normally caused by the decrease in a level of phosphatase or the presence of phosphatase deletions. In addition, the phosphorylation processes in the activation of AKT occur relatively faster than the degradation processes. This condition leads us to assume that

the degradation rate of AKT can be neglected. Therefore, we consider the following system of ODEs:

$$
\begin{aligned}
& \frac{d x_{1}}{d t}=k_{0}+b x_{5}-d_{1} x_{1}, \\
& \frac{d x_{2}}{d t}=a_{2}-\frac{k_{2} x_{1} x_{2}^{2}}{K_{2}^{2}+x_{2}^{2}}, \\
& \frac{d x_{3}}{d t}=\frac{k_{2} x_{1} x_{2}^{2}}{K_{2}^{2}+x_{2}^{2}}-d_{3} x_{3}, \\
& \frac{d x_{4}}{d t}=\left(x_{4}-\Lambda\right)\left(\left(p-m x_{4}\right)-\frac{k_{4} x_{3} x_{4}^{2}}{K_{4}^{3}+x_{4}^{3}}\right), \\
& \frac{d x_{5}}{d t}=\frac{k_{4} x_{3} x_{4}^{2}\left(x_{4}-\Lambda\right)}{K_{4}^{3}+x_{4}^{3}}-d_{5} x_{5} .
\end{aligned}
$$


The system (1) describes the interactions between PIP3, AKT, phosphorylated AKT (AKTp), FOXO3a and phosphorylated FOXO3a (FOXO3ap) in the PI3K/AKT pathway denoted by $x_{1}, x_{2}, x_{3}, x_{4}$ and $x_{5}$, respectively. All the parameter values are positive constants, with the following interpretation: $k_{0}$ is the formation PIP3 by PI3K, $b$ is the increase activation PIP3 by FOXO3ap, $a_{2}$ is the AKT production rate, $k_{2}$ and $k_{4}$ are the constant rates of $\mathrm{AKT}$ and FOXO3a phosphorylation, respectively, $K_{2}$ and $K_{4}$ are the Michaelis constants of AKT and FOXO3a phosphorylation, respectively, $\Lambda$ is the rest of FOXO3a in the nucleus, $p$ is the FOXO3a production rate, $m$ is the degradation rate of FOXO3a by 14-3-3 protein, $d_{1}, d_{3}$ and $d_{5}$ are the degradation rates of PIP3, AKTp and FOXO3ap, respectively. Next, we want to determine the equilibrium point of the system (1). In addition, we note that in a healthy cell, there are no FOXO3a in the cytoplasm so that the AML-free equilibrium point indicates the zero concentration of FOXO3ap. Therefore, we find that the system (1) admits the AML-free equilibrium point

$$
E_{0}=\left(\frac{k_{0}}{d_{1}}, \frac{K_{2} \sqrt{a_{2} d_{1}}}{\sqrt{k_{0} k_{2}-a_{2} d_{1}}}, \frac{a_{2}}{d_{3}}, \Lambda, 0\right)
$$

and the other equilibrium point

$$
E_{1 i}^{*}=\left(x_{1 i}^{*}, x_{2 i}^{*}, x_{3 i}^{*}, x_{4 i}^{*}, x_{5 i}^{*}\right),
$$

where

$$
\begin{aligned}
& x_{1 i}^{*}=\frac{k_{0}}{d_{1}}+\frac{b k_{4} a_{2} x_{4 i}^{* 2}\left(x_{4 i}^{*}-\Lambda\right)}{d_{1} d_{3} d_{5}\left(K_{4}^{3}+x_{4 i}^{* 3}\right)} \\
& x_{2 i}^{*}=\frac{K_{2} \sqrt{a_{2} d_{1} d_{3} d_{5}\left(K_{4}^{3}+x_{4 i}^{* 3}\right)}}{\sqrt{\left(k_{0} k_{2}-a_{2} d_{1}\right) d_{3} d_{5}\left(K_{4}^{3}+x_{4 i}^{* 3}\right)+b k_{2} k_{4} a_{2} x_{4 i}^{* 2}\left(x_{4 i}^{*}-\Lambda\right)}}, \\
& x_{3 i}^{*}=\frac{a_{2}}{d_{3}}
\end{aligned}
$$




$$
x_{5 i}^{*}=\frac{k_{4} a_{2} x_{4 i}^{* 2}\left(x_{4 i}^{*}-\Lambda\right)}{d_{3} d_{5}\left(K_{4}^{3}+x_{4 i}^{* 3}\right)}
$$

and $x_{4 i}^{*}, i=1,2,3,4$ is a positive solution of the following equation:

$$
x_{4}^{4}+c_{3} x_{4}^{3}+c_{2} x_{4}^{2}+c_{1} x_{4}+c_{0}=0
$$

with

$$
c_{3}=-\frac{p}{m}, \quad c_{2}=\frac{a_{2} k_{4}}{m d_{3}}, \quad c_{1}=K_{4}^{3}, \quad c_{0}=-\frac{p K_{4}^{3}}{m} .
$$

Now, we focus on the AML-free equilibrium point $E_{0}$ and investigate the occurrence of both backward and forward bifurcations. Note that the equilibrium point $E_{0}$ always exists whenever $k_{0} k_{2}-a_{2} d_{1}>0$. For stability of the equilibrium point $E_{0}$, we introduce the threshold value $\Gamma$ :

$$
\Gamma=\frac{p}{m \Lambda+\frac{k_{4} a_{2} \Lambda^{2}}{d_{3}\left(K_{4}^{3}+\Lambda^{3}\right)}}
$$

and state the following theorem.

Theorem 2.1. The AML-free equilibrium point $E_{0}$ is locally asymptotically stable if $\Gamma<1$ and unstable if $\Gamma>1$.

Proof. The eigenvalues of Jacobian matrix of system (1) at $E_{0}=$ $\left(\frac{k_{0}}{d_{1}}, \frac{K_{2} \sqrt{a_{2} d_{1}}}{\sqrt{k_{0} k_{2}-a_{2} d_{1}}}, \frac{a_{2}}{d_{3}}, \Lambda, 0\right)$ are $\lambda_{1}=-\frac{2 \sqrt{a_{2} d_{1}} \sqrt{\left(k_{0} k_{2}-a_{2} d_{1}\right)^{3}}}{k_{0} k_{2} K_{2} d_{1}}, \lambda_{2}=-d_{1}$, $\lambda_{3}=-d_{3}, \quad \lambda_{4}=p-m \Lambda-\frac{k_{4} a_{2} \Lambda^{2}}{d_{3}\left(K_{4}^{3}+\Lambda^{3}\right)}$ and $\lambda_{5}=-d_{5}$. We have four eigenvalues that are always strictly negative. We can see that $\lambda_{4}=p$ $-m \Lambda-\frac{k_{4} a_{2} \Lambda^{2}}{d_{3}\left(K_{4}^{3}+\Lambda^{3}\right)}$, can be either positive or negative. We found that all 
eigenvalues are negative if $p<m \Lambda+\frac{k_{4} a_{2} \Lambda^{2}}{d_{3}\left(K_{4}^{3}+\Lambda^{3}\right)}$ or $\Gamma<1$ and $E_{0}$ is locally asymptotically stable, and if $\Gamma>1$, then there is one positive eigenvalue, so that $E_{0}$ is unstable. This completes the proof.

From (7), we find that $\Gamma=1$ iff $p=p^{*}=m \Lambda+\frac{k_{4} a_{2} \Lambda^{2}}{d_{3}\left(K_{4}^{3}+\Lambda^{3}\right)}$ and $E_{0}$ is locally asymptotically stable if $p<p^{*}$, whereas it loses its stability if $p>p^{*}$. Furthermore, in the next section, we will perform that the system (1) can undergo the forward or backward bifurcation at $p=p^{*}$.

\section{Bifurcation Analysis}

In this section, we will make use of the bifurcation method introduced in [8] to derive the sufficient conditions for both backward and forward bifurcation scenarios. We then claim the following theorem.

Theorem 3.1. The system (1) at $\Gamma=1$ exhibits:

a. Backward bifurcation whenever:

$$
\left\{\begin{array}{l}
\Lambda^{3}-2 K_{4}^{3}>0 \\
\frac{k_{4} a_{2} \Lambda\left(\Lambda^{3}-2 K_{4}^{3}\right)}{d_{3}\left(K_{4}^{3}+\Lambda^{3}\right)^{2}}-m>0
\end{array}\right.
$$

b. Forward bifurcation whenever:

$$
\Lambda^{3}-2 K_{4}^{3}<0
$$

or

$$
\left\{\begin{array}{l}
\Lambda^{3}-2 K_{4}^{3}>0 \\
\frac{k_{4} a_{2} \Lambda\left(\Lambda^{3}-2 K_{4}^{3}\right)}{d_{3}\left(K_{4}^{3}+\Lambda^{3}\right)^{2}}-m<0 .
\end{array}\right.
$$


Proof. From the last section, we find that $\Gamma=1$ associated with $p=p^{*}=m \Lambda+\frac{k_{4} a_{2} \Lambda^{2}}{d_{3}\left(K_{4}^{3}+\Lambda^{3}\right)}$, and eigenvalues of the Jacobian matrix at $\left(E_{0}, p^{*}\right)$ as $\lambda_{1}=-\frac{2\left(k_{0} k_{2}-a_{2} d_{1}\right) \sqrt{a_{2} d_{1}\left(k_{0} k_{2}-a_{2} d_{1}\right)}}{k_{0} k_{2} K_{2} d_{1}}, \lambda_{2}=-d_{1}, \lambda_{3}=-d_{3}$, $\lambda_{4}=0$ and $\lambda_{5}=-d_{5}$

Obviously, $\lambda_{4}=0$ is a single zero eigenvalue and all other eigenvalues are real and negative. Thus, $E_{0}$ is a nonhyperbolic equilibrium point and system (1) can undergo a bifurcation at $p=p^{*}=m \Lambda+\frac{k_{4} a_{2} \Lambda^{2}}{d_{3}\left(K_{4}^{3}+\Lambda^{3}\right)}$ (or equivalently at $\Gamma=1$ ). Now, we will determine the eigenvector associated with the zero eigenvalues $\lambda_{4}=0$.

Let $\mathbf{w}=\left(w_{1}, w_{2}, w_{3}, w_{4}, w_{5}\right)^{T}$ be the right eigenvector of the matrix $J\left(E_{0}, p^{*}\right)$. Then

$$
\left\{\begin{array}{l}
-d_{1} w_{1}+b w_{5}=0, \\
-\frac{a_{2} d_{1}}{k_{0}} w_{1}-\frac{2\left(k_{0} k_{2}-a_{2} d_{1}\right) \sqrt{a_{2} d_{1}\left(k_{0} k_{2}-a_{2} d_{1}\right)}}{K_{2} d_{1} k_{0} k_{2}} w_{2}=0, \\
\frac{a_{2} d_{1}}{k_{0}} w_{1}+\frac{2\left(k_{0} k_{2}-a_{2} d_{1}\right) \sqrt{a_{2} d_{1}\left(k_{0} k_{2}-a_{2} d_{1}\right)}}{K_{2} d_{1} k_{0} k_{2}} w_{2}-d_{3} w_{3}=0, \\
\frac{k_{4} a_{2} \Lambda^{2}}{K_{4}^{3}+\Lambda^{3}} w_{4}-d_{5} w_{5}=0,
\end{array}\right.
$$

so that

$$
\mathbf{w}=\left(1,-\frac{a_{2} d_{1}^{2} K_{2} k_{2}}{2\left(k_{0} k_{2}-a_{2} d_{1}\right) \sqrt{a_{2} d_{1}\left(k_{0} k_{2}-a_{2} d_{1}\right)}}, 0, \frac{d_{1} d_{5}\left(K_{4}^{3}+\Lambda^{3}\right)}{k_{4} a_{2} \Lambda^{2} b}, \frac{d_{1}}{b}\right)^{T} .
$$


It is easy to show that the left eigenvector of the matrix $J\left(E_{0}, p^{*}\right)$ satisfying $\mathbf{v} \cdot \mathbf{w}=1$ is given by

$$
\mathbf{v}=\left(0,0,0, \frac{k_{4} a_{2} \Lambda^{2} b}{d_{1} d_{5}\left(K_{4}^{3}+\Lambda^{3}\right)}, 0\right) .
$$

Furthermore, we define the coefficients $a$ and $b$ :

$$
a=\sum_{k, i, j=1}^{5} v_{k} w_{i} w_{j} \frac{\partial^{2} f_{k}}{\partial x_{i} \partial x_{j}}\left(E_{0}, p^{*}\right), \quad b=\sum_{k, i=1}^{5} v_{k} w_{i} \frac{\partial^{2} f_{k}}{\partial x_{i} \partial p}\left(E_{0}, p^{*}\right) .
$$

Then considering the components of the right and left eigenvectors $w_{3}=0$ and $v_{1}=v_{2}=v_{3}=v_{5}=0$, it follows that

$$
\begin{aligned}
a= & v_{4} w_{1}^{2} \frac{\partial^{2} f_{4}}{\partial x_{1}^{2}}\left(E_{0}, p^{*}\right)+v_{4} w_{1} w_{2} \frac{\partial^{2} f_{4}}{\partial x_{1} \partial x_{2}}\left(E_{0}, p^{*}\right) \\
& +v_{4} w_{1} w_{2} \frac{\partial^{2} f_{4}}{\partial x_{1} \partial x_{4}}\left(E_{0}, p^{*}\right)+v_{4} w_{1} w_{5} \frac{\partial^{2} f_{4}}{\partial x_{1} \partial x_{5}}\left(E_{0}, p^{*}\right) \\
& +v_{4} w_{2} w_{1} \frac{\partial^{2} f_{4}}{\partial x_{2} \partial x_{1}}\left(E_{0}, p^{*}\right)+v_{4} w_{2}^{2} \frac{\partial^{2} f_{4}}{\partial x_{2}^{2}}\left(E_{0}, p^{*}\right) \\
& +v_{4} w_{2} w_{4} \frac{\partial^{2} f_{4}}{\partial x_{2} \partial x_{4}}\left(E_{0}, p^{*}\right)+v_{4} w_{2} w_{5} \frac{\partial^{2} f_{4}}{\partial x_{2} \partial x_{5}}\left(E_{0}, p^{*}\right) \\
& +v_{4} w_{4} w_{1} \frac{\partial^{2} f_{4}}{\partial x_{4} \partial x_{1}}\left(E_{0}, p^{*}\right)+v_{4} w_{4} w_{2} \frac{\partial^{2} f_{4}}{\partial x_{4} \partial x_{2}}\left(E_{0}, p^{*}\right) \\
& +v_{4} w_{4}^{2} \frac{\partial^{2} f_{4}}{\partial x_{4}^{2}}\left(E_{0}, p^{*}\right)+v_{4} w_{4} w_{5} \frac{\partial^{2} f_{4}}{\partial x_{4} \partial x_{5}}\left(E_{0}, p^{*}\right) \\
& +v_{4} w_{5} w_{1} \frac{\partial^{2} f_{4}}{\partial x_{5} \partial x_{1}}\left(E_{0}, p^{*}\right)+v_{4} w_{5} w_{2} \frac{\partial^{2} f_{4}}{\partial x_{5} \partial x_{2}}\left(E_{0}, p^{*}\right) \\
& +v_{4} w_{5} w_{4} \frac{\partial^{2} f_{4}}{\partial x_{5} \partial x_{4}}\left(E_{0}, p^{*}\right)+v_{4} w_{5}^{2} \frac{\partial^{2} f_{4}}{\partial x_{5}^{2}}\left(E_{0}, p^{*}\right)
\end{aligned}
$$


and

$$
b=v_{4} w_{1} \frac{\partial^{2} f_{4}}{\partial x_{1} \partial p}+v_{4} w_{2} \frac{\partial^{2} f_{4}}{\partial x_{2} \partial p}+v_{4} w_{4} \frac{\partial^{2} f_{4}}{\partial x_{4} \partial p}+v_{4} w_{5} \frac{\partial^{2} f_{4}}{\partial x_{5} \partial p}
$$

In view of (11), (12) and $f_{4}=\left(x_{4}-\Lambda\right)\left(p-m \Lambda-\frac{k_{4} a_{2} x_{3} x_{4}^{2}}{K_{4}^{3}+\Lambda^{3}}\right)$, we then get:

$$
a=\frac{2 d_{1} d_{5}\left(K_{4}^{3}+\Lambda^{3}\right)}{b k_{4} a_{2} \Lambda^{2}}\left(\frac{k_{4} a_{2} \Lambda\left(\Lambda^{3}-2 K_{4}^{3}\right)}{d_{3}\left(K_{4}^{3}+\Lambda^{3}\right)^{2}}-m\right)
$$

and

$$
b=1 \text {. }
$$

The coefficient $b$ is always positive so that, by applying Theorem 4 in [8], we may conclude that the system (1) exhibits a backward bifurcation if $\frac{k_{4} a_{2} \Lambda\left(\Lambda^{3}-2 K_{4}^{3}\right)}{d_{3}\left(K_{4}^{3}+\Lambda^{3}\right)^{2}}-m>0$ and a forward bifurcation if $\frac{k_{4} a_{2} \Lambda\left(\Lambda^{3}-2 K_{4}^{3}\right)}{d_{3}\left(K_{4}^{3}+\Lambda^{3}\right)^{2}}$ $-m<0$.

Hence, for the existence of a backward or a forward bifurcation, we have the following conditions: if $\Lambda^{3}-2 K_{4}^{3}>0$ and $\frac{k_{4} a_{2} \Lambda\left(\Lambda^{3}-2 K_{4}^{3}\right)}{d_{3}\left(K_{4}^{3}+\Lambda^{3}\right)^{2}}-m>0$, then the system (1) exhibits a backward bifurcation at $\Gamma=1$, whereas if: (i) $\Lambda^{3}-2 K_{4}^{3}<0$ or (ii) $\Lambda^{3}-2 K_{4}^{3}>0$ and $\frac{k_{4} a_{2} \Lambda\left(\Lambda^{3}-2 K_{4}^{3}\right)}{d_{3}\left(K_{4}^{3}+\Lambda^{3}\right)^{2}}-m<0$, then the system (1) exhibits a forward bifurcation at $\Gamma=1$. This completes the proof.

\section{Numerical Simulations}

In this section, we provide a numerical simulation to verify the theoretical results obtained in the previous section. In the first scenario, we 
consider the set of parameter values:

$$
\begin{aligned}
& k_{0}=0.01, b=0.0083, d_{1}=0.033, a_{2}=0.09, d_{3}=0.09, \Lambda=0.2, p=0.2, \\
& m=0.28, k_{2}=1, K_{2}=0.1, k_{4}=0.04, K_{4}=0.1, d_{5}=0.125
\end{aligned}
$$

With these parameter values, we have the AML-free equilibrium point $E_{0}=(0.3030303,0.64998,1,0.2,0)$, and the condition (8) in Theorem 3.1 is satisfied, that is $\Lambda^{3}-2 K_{4}^{3}=0.006>0$, and $\frac{k_{4} a_{2} \Lambda\left(\Lambda^{3}-2 K_{4}^{3}\right)}{d_{3}\left(K_{4}^{3}+\Lambda^{3}\right)^{2}}-m=$ $0.3125>0$. If the equilibrium point $E_{0}$ is continued with respect to a parameter $p$, then the system (1) exhibits a backward bifurcation at $p=p^{*}=0.233778$. The saddle-node bifurcation value is $p_{2}=$ 0.209575955 (see Figure 1). Figure 1 shows that there is an equilibrium point $E_{11}$ which is stable besides AML-free equilibrium point $E_{0}$ when $\Gamma<1$. This indicates that to eliminate the disease, it might not be sufficient to reduce $\Gamma$ below 1 . We can see from Figure 1 that to eliminate the disease, $p$ must be below 0.2095759556, where at $p_{2}=0.209575955, \Gamma_{2}=$ 0.8964750952. In the interval $0.8964750952<\Gamma<1$, we have three equilibrium points, that is an unstable equilibrium point $E_{12}$, and stable equilibrium points $E_{11}$ and $E_{0}$.

In the second scenario, by choosing $k_{2}=2, k_{4}=0.12$ and $K_{4}=0.2$, and keeping the other parameter values as before, we have $E_{0}=$ $(0.3030303,0.041761,1,0.2,0)$ and the condition (9) of Theorem 3.1 is satisfied, that is $\Lambda^{3}-2 K_{4}^{3}=-0.008<0$. In this scenario, forward bifurcation occurs at $p=p^{*}=0.3560000356$. Figure 2 shows that when $p<0.3560000356$ or equivalent $\Gamma<1$, there is only one stable equilibrium point, that is AML-free equilibrium point $E_{0}$. Hence, to eliminate the AML disease or to prevent AML disease outbreaks, we can identify the value parameter $p$ necessary to reduce $\Gamma$ below 1 . In this case, we also find a 
saddle-node bifurcation at two points, which indicates the occurrence of an unusual phenomenon of forward bifurcation with hysteresis. The saddlenode bifurcation values are $p_{1}=0.36082380695$ and $p_{2}=0.39276755124$ (see Figure 2). Figure 2 shows that the system (1) exhibits a hysteresis effect where two stable equilibria $E_{11}$ and $E_{12}$ coexist when $\Gamma>1$ if $p_{1}<p^{*}<p_{2}$.

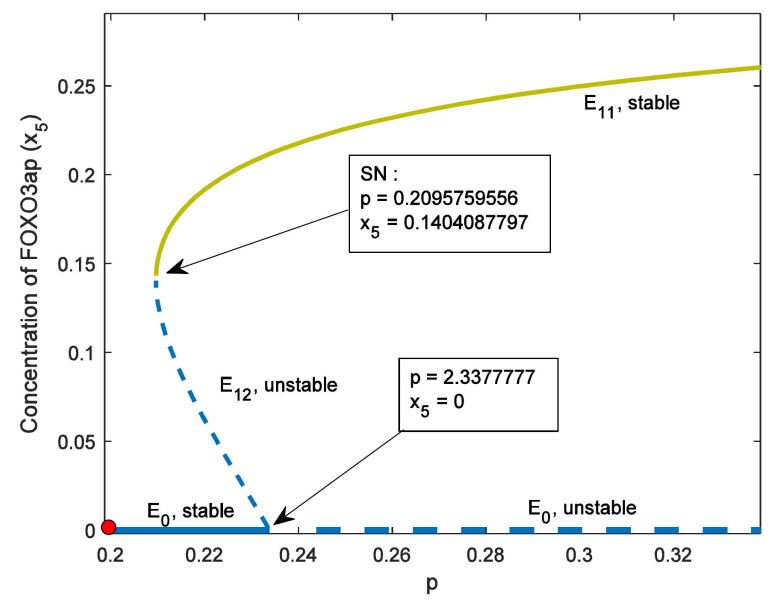

Figure 1. Backward bifurcation at $p=p^{*}=0.233778$.

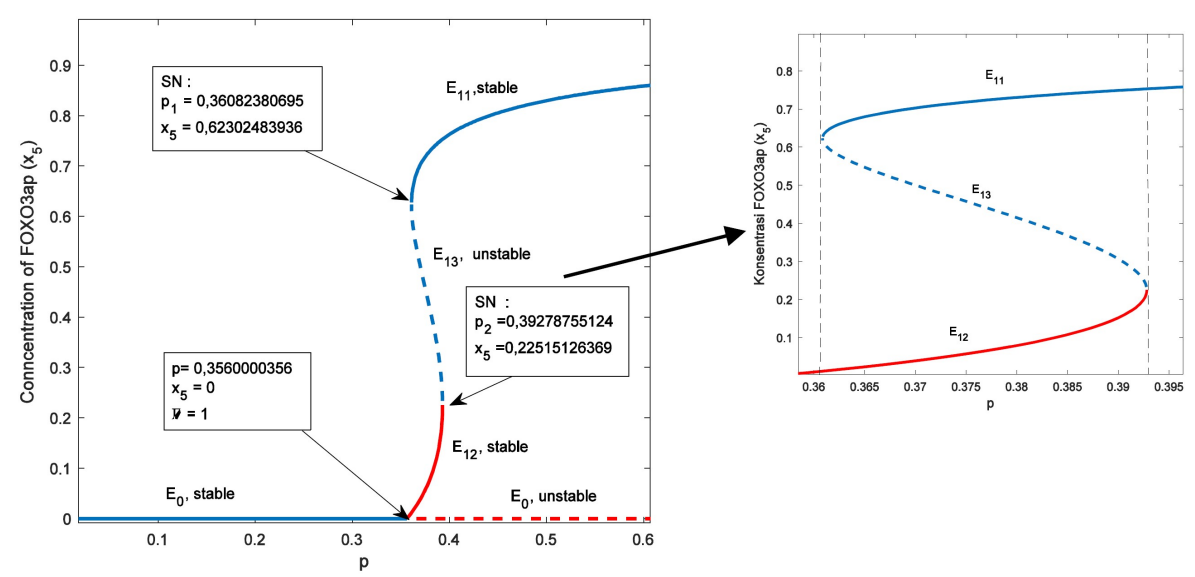

Figure 2. Forward bifurcation at $p=p^{*}=0.3560000356$. 


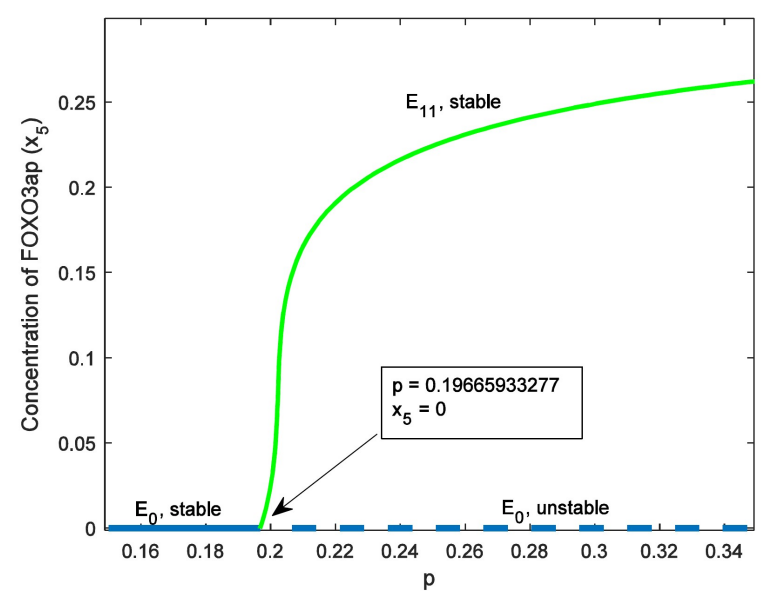

Figure 3. Forward bifurcation at $p=p^{*}=1.9665933277$.

As shown in Figure 2, if $0.3560000356<p<0.36082380695$, then there is only one stable equilibrium point $E_{12}$ which has low FOXO3ap concentration and if $p>0.39276755124$, then there is only one stable equilibrium $E_{11}$ which has higher FOXO3a concentration. In the last scenario, we choose $K_{4}=0.15$ keeping the other parameters as in the first scenario. With these parameter values, we have $\Lambda^{3}-2 K_{4}^{3}=0.00125>0$ and $\frac{k_{4} a_{2} \Lambda\left(\Lambda^{3}-2 K_{4}^{3}\right)}{d_{3}\left(K_{4}^{3}+\Lambda^{3}\right)^{2}}-m=-0.2027146<0$, so that the condition (10) in Theorem 3.1 is satisfied. The system (1) exhibits a forward bifurcation at $p=p^{*}=0.196665933277$ (see Figure 3).

As shown in Figure 3, before the bifurcation point, there is only one stable AML-free disease equilibrium point $E_{0}$, and after the bifurcation point, there is one stable equilibrium point $E_{11}$ and one unstable AML-free disease equilibrium $E_{0}$. Hence, it is possible to conclude that, to eradicate the AML disease, the value of $p$ should be below the bifurcation point $p=p^{*}=0.196665933277$. 


\section{Discussion and Conclusion}

In this paper, we considered a mathematical model of the PI3K/AKT pathway in acute myeloid leukemia. We found the threshold value $\Gamma$, so that the AML-free equilibrium point is stable when $\Gamma<1$ and unstable when $\Gamma>1$. In the previous section, we performed the conditions such that system (1) undergoes a backward or forward bifurcation at $\Gamma=1$. These conditions are depicted in (8)-(10).

Our analysis suggests that FOXO3a plays an important role in AML disease and may be used as a gauge in distinguishing between the occurrences of backward or forward bifurcation. Observe that the Michaelis constant of AKT phosphorylation $K_{4}$ is the concentration of FOXO3a for half-maximal phosphorylation FOXO3a by AKT and $\Lambda$ is the rest of FOXO3a in the nucleus. According to Theorem 3.1, forward bifurcation occurs if $\Lambda^{3}-2 K_{4}^{3}<0$ or $\Lambda-2^{\frac{1}{3}} K_{4}<0$. This forward bifurcation results in the existence of AML equilibrium point that is stable after passing the bifurcation point. Furthermore, AML treatment for this case can be done by reducing the rate of $\mathrm{FOXO} 3$ a production, so that $\Gamma$ below 1 . However, in this case, a hysteresis phenomenon occurs. At the time of the occurrence of this phenomenon, medical steps cannot be determined for AML treatment because when hysteresis occurs, the conditions of proteins are more difficult to observe.

If $\Lambda^{3}-2 K_{4}^{3}>0$, from Theorem 3.1 , the system (1) can undergo backward and forward bifurcations. This occurrence depends on $\frac{k_{4} a_{2} \Lambda\left(\Lambda^{3}-2 K_{4}^{3}\right)}{d_{3}\left(K_{4}^{3}+\Lambda^{3}\right)^{2}}-m$ which can medically interpret as a difference between the component of phosphorylated FOXO3a that translocates from the nucleus to the cytoplasm and the FOXO3a degradation rate. The backward bifurcation occurs if the factors or components cause FOXO3a 
translocation to the cytoplasm greater than the FOXO3a degradation rate, whereas, if the FOXO3a degradation rate is larger than the component that causes translocation of FOXO3a to the cytoplasm, there will be a forward bifurcation. Based on the bifurcation diagram, in this case, the hysteresis phenomenon does not appear, so from the medical point of view, the AML treatment strategy is easier to determine. One component that causes translocation FOXO3a from the nucleus to the cytoplasm in the expression is the concentration of AKT phosphorylation, which is expressed as $\frac{a_{2}}{d_{3}}$. Therefore, inhibiting the concentration of AKT phosphorylation can also be done to reduce the translocation of FOXO3a from the nucleus to the cytoplasm so that the occurrence of backward bifurcation can be avoided. However, further research is needed.

Finally, these results suggest that, in AML treatment, the targeted therapy can be done by targeting the FOXO3a. This is also in accordance with Zhang et al. [4], which states that leukemia cells depend on the release of protein, in this case, FOXO3a from the nucleus through phosphorylation and the return of protein to the nucleus in the presence of normal function of the tumor suppressor. Therefore, targeting FOXO3a can be combined with target therapy on FLT3. The combination of target therapy is expected to restore normal function PI3K/AKT pathway so that the development of AML cells can be controlled.

\section{Acknowledgments}

The authors would like to express their gratitude to the editors and anonymous reviewers for useful comments and suggestions. Also, the special thanks to the Cancer Modeling Team UGM for the discussions during the research.

This work was supported by the Ministry of Research and Higher Education of Indonesia. 


\section{References}

[1] H. Dohner, E. Estey, D. Grimwade et al., Diagnosis and management of AML in adults: 2017 ELN recommendations from an international expert panel, Blood 129(4) (2017), 424-447.

[2] S. Kayser and M. J. Levis, Advances in targeted therapy for acute myeloid leukemia, Br. J. Haematol. 180(4) (2018), 484-500.

[3] M. Larrosa-Garcia and M. R. Baer, FLT3 inhibitor in acute myeloid leukemia: current status and future directions, Mol. Cancer Ther. 16(6) (2017), 991-1001.

[4] W. Zhang, C. Ly, J. Ishizawa, H. Mu, V. Ruvolo, S. Shacham, N. Daver and M. Andreeff, Combinatorial targeting of XPO1 and FLT3 exert synergistic antileukemia effects through induction of differentiation and apoptosis in FLT3mutated acute myeloid leukemia: from concept to clinical trial, Haematologica 103(10) (2018), 1642-1653.

[5] M. Levis, Midostaurin approved for FLT3 mutated AML, Blood 129(28) (2017), 3403-3406.

[6] Y. A. Adi, F. Adi-Kusumo, L. Aryati and M. S. Hardianti, A dynamic model of PI3K/AKT pathways in acute myeloid leukemia, J. Appl. Math. 2018, Art. ID 2983138, 9 pp.

[7] M. Andreeff, Targeted Therapy of Acute Myeloid Leukemia, Springer, New York, NY, USA, 2015.

[8] C. Castillo-Chavez and B. Song, Dynamical model of tuberculosis and their applications, Math. Biosci. Eng. 1 (2004), 361-404.

[9] S. M. Garba, A. B. Gumel and M. R. Abu Bakar, Backward bifurcations in dengue transmission dynamics, Math. Biosci. 215 (2008), 11-25.

[10] B. Buonomo and D. Lacitignola, On the backward bifurcation of vaccination model with nonlinear incidence, Nonlinear Anal. Model. Control 16(1) (2011), $30-46$.

[11] F. Brauer, Backward bifurcations in simple vaccination/treatment models, Journal of Biological Dynamics 5(5) (2011), 410-418. http://dx.doi.org/10.1080/ 17513758.2010 .510584 .

[12] L. C. Mpande, D. Kajunguri and E. A. Mpolya, Modeling and stability analysis for measles metapopulation model with vaccination, Appl. Comput. Math. 4(6) (2015), 431-444. 
[13] D. H. Knipl and G. Rost, Backward bifurcation in SIVS model with immigration of non-infectives, Biomath. 2 (2013), 1-14.

[14] I. M. Wangari and L. Stone, Backward bifurcation and hysteresis in models of recurrent tuberculosis, PLOS ONE 13(3) (2018).

[15] S. Wiggins, Introduction to Applied Nonlinear Dynamical Systems and Chaos, Springer-Verlag, New York, Inc., 2003.

[16] R. S. Nho and P. Hergert, FOXO3a and disease progression, World J. Biol. Chem. 5(3) (2014), 346-354. 